\title{
Evaluación entre iguales en entornos de educación superior online mediante el taller de Moodle. Estudio de caso
}

\author{
Ana Cristina García, Manuel Gil-Mediavilla, Ildefonso Álvarez y María de los Ángeles Casares \\ Universidad Isabel I, Facultad de Humanidades y Ciencias Sociales, Calle Fernán González 76. 09003. Burgos - \\ España. (correo-e: anacristina.garcia@ui1.es; manuel.gil@ui1.es; ildefonso.alvarez@ui1.es; \\ mariadelosangeles.casares@ui1.es)
}

Recibido Ago. 28, 2019; Aceptado Oct. 22, 2019; Versión final Nov. 13, 2019, Publicado Abr. 2020

\section{Resumen}

A través de este estudio se busca validar un instrumento que recoge la opinión del alumnado acerca del uso del Taller de Moodle como herramienta para facilitar la evaluación entre iguales en la educación superior online. El estudio estadístico se basó en el análisis factorial y la determinación del coeficiente de fiabilidad de Cronbach. Los resultados muestran que el instrumento es fiable $(\alpha=0.878)$, extrayéndose un solo factor que permite interpretar las opiniones de los estudiantes ante el uso de la herramienta para la evaluación entre iguales. Por lo tanto, el instrumento propuesto se puede utilizar con fines de investigación, posibilitando el desarrollo de posteriores estudios que validen empíricamente el uso del Taller de Moodle. También, permitirá continuar profundizando en otros factores que pueden influir en la mejora de los procesos de evaluación entre iguales en la educación online.

\section{Peer assessment in higher education using Moodle workshop. A case study}

\begin{abstract}
This study seeks to validate a tool which gathers students' opinion about the use of Moodle Workshop as a means of evaluating peer assessment in online higher education. The statistical study was based on factor analysis and on determining Cronbach's Alpha. Results show that the tool is reliable $(\alpha=0.878)$ and only one factor with satisfactory values was extracted, allowing for the interpretation of students' opinion regarding the peer assessment tool. Therefore, the proposed method can be used for research purposes, enabling further studies which will empirically validate the use of Moodle Workshop. At the same time, it will allow deepening the knowledge of other factors that may contribute to improving peer assessment processes in online education.
\end{abstract}

Keywords: evaluation; online teaching; higher education; information and communication technologies; educational innovation 


\section{INTRODUCCIÓN}

La evaluación en entornos virtuales es un proceso complejo, incluso un reto mayor que el propio proceso de aprendizaje, convirtiéndose en uno de los pilares fundamentales que aseguran el éxito de cualquier acción formativa. Sin embargo. cada día surgen nuevos recursos tecnológicos que facilitan el desarrollo de una evaluación formativa enfocada no solo en el producto, sino también el proceso de enseñanza-aprendizaje (Troy y Bulgakov, 2013; Cook y Ellaway, 2015; De la Hoz et al., 2015). La evaluación impacta en todo el proceso de aprendizaje, por lo tanto, debe desarrollarse analizando todos los elementos y variables que pueden incidir en el curso, como el diseño instruccional del curso, las características de la plataforma educativa que utilicemos, el grado de implicación del alumnado en el proceso de enseñanza aprendizaje y las funciones del profesorado. Tal como señala Barberà (2016) "...la evaluación no es solamente evaluación del aprendizaje, sino que es también evaluación para el aprendizaje" (pp.5), convirtiendo la retroalimentación, el diálogo y la reflexión, en elementos fundamentales de la práctica educativa (Ho et al., 2018). Partiendo de esta apreciación, se debe analizar el contexto, así como los avances tecnológicos para orientar la práctica docente al desarrollo de experiencias que promuevan una evaluación más participativa e integral. La práctica evaluativa revela la importancia de explorar diferentes enfoques para obtener una mayor calidad en el proceso. En el caso de los entornos virtuales, las tecnologías ofrecen diversas oportunidades, gracias a los aportes que ha realizado en el campo de la evaluación (Obura et al., 2018; Wang et al., 2006; Cassady y Gridley, 2005; Barberà, 2016).

Al revisar la literatura se pueden encontrar distintas estrategias de evaluación como la resolución de problemas, los estudios de caso. Proyectos, debates, la autoevaluación, evaluación entre iguales, el uso de portafolios electrónicos, los exámenes con retroalimentación automática, los ensayos, trabajos colaborativos y simulaciones (Ibarra et al., 2012; Guardia y Sangrá, 2005). A simple vista se puede observar que la mayoría de las estrategias señaladas anteriormente se aplican en el aprendizaje presencial, sin embargo, el reto al cual se enfrentan las instituciones es cómo aprovechar las ventajas que nos ofrecen las tecnologías, para transformar de forma creativa la aplicación de estas al aprendizaje online. De acuerdo con algunos estudios, un entorno que integra el aprendizaje en línea y el uso de recursos de evaluación formativa ofrece grandes beneficios para el alumnado (Wang et al., 2006; Cassady y Gridley, 2005). Se pueden señalar diversas herramientas que apoyan la evaluación en el aprendizaje online, pero de acuerdo con el objetivo del estudio, nos centraremos en las oportunidades que ofrece el módulo de Taller que posee Moodle.

Moodle, como sistema de gestión de aprendizaje, ofrece múltiples ventajas para facilitar el trabajo colaborativo, la participación e interacción en el aula, así como el uso de distintos módulos con actividades y recursos centrados en el discente como principal protagonista de su aprendizaje (Jenaro et al., 2018). Entre estos módulos ofrece el Taller. que facilita llevar a cabo procesos de evaluación entre iguales. A través del Taller se puede recopilar cualquier contenido digital, revisar y evaluar el trabajo de los estudiantes desde un rol activo, es decir, integrando su participación en el proceso de evaluación (Deepak, 2017; Cordero y Caballero, 2015). Existen diversas investigaciones que avalan los beneficios que ofrece la evaluación entre iguales como estrategia para el aprendizaje (Acedo y Ruiz-Cabestre, 2011; Kiliç, 2016; Thawabieh, 2017; Kishwar et al., 2015; Siow, 2015), sin embargo, se encuentran pocos estudios que validen empíricamente el uso del Taller como herramienta para aplicar dicha estrategia (Strang, 2015). Se han encontrado experiencias que muestran su utilidad (Gallego et al., 2017), al igual que casos en que los usuarios lo consideran poco valioso en comparación con otros recursos que ofrece Moodle (Deepak, 2017).

Con el fin de evidenciar la eficacia del uso de esta herramienta tecnológica para la evaluación del aprendizaje, se elaboró un cuestionario que permitiera recoger la opinión del alumnado sobre las ventajas y desventajas del Taller, al igual que valorar las implicaciones que puede suponer el uso de esta sobre su aprendizaje. Para ello se llevó a cabo un estudio instrumental para analizar las propiedades psicométricas del cuestionario en el contexto de la asignatura La Accción Tutorial en E-learning del Máster en Diseño Tecnopedagógico. Se examinó igualmente la estructura interna y la consistencia del cuestionario, la homogeneidad y los estadísticos descriptivos de los ítems, así como las frecuencias de sujetos que seleccionaron cada una de las categorías. Esta información permitirá organizar los datos para interpretar las respuestas de los estudiantes de forma objetiva. así como disponer de un instrumento validado que posibilite el desarrollo de nuevas investigaciones y el establecimiento de mejores prácticas dentro del campo del aprendizaje basado en la tecnología.

\section{METODOLOGÍA}

Para la selección de los participantes en el estudio se llevó a cabo un muestreo no probabilístico causal o incidental. El cuestionario se envió a los 110 alumnos, del curso 2017/2018 matriculados en la asignatura La Acción Tutorial en E-learning, del Máster en Diseño Tecnopedagógico de la Universidad Isabel I. De esta forma se pudo trabajar con un grupo homogéneo en cuanto a variables de interés como el curso recibido, el docente que lo impartía y la temporalización seguida. Se recibieron un total de 34 respuestas voluntarias y debidamente cumplimentadas, lo que supone un $31 \%$ del grupo que realizó el curso. Estos datos permitirán comprender el significado de la experiencia de la evaluación entre iguales para los alumnos implicados, 
mejorar la praxis educativa y validar un constructo que permita continuar profundizando en los procesos de evaluación apoyados por tecnología.

El cuestionario, elaborado ex profeso para esta investigación, está compuesto por 15 ítems, que permiten recoger la opinión de los estudiantes acerca del uso de la herramienta Taller y el impacto que tiene para su aprendizaje. Los ítems se puntuaron mediante una escala tipo Likert de 5 puntos (Total Desacuerdo=1, Desacuerdo $=2$, Indiferente $=3$, Acuerdo $=4$ y Total Acuerdo $=5$ ). La experiencia se estructuró a través de la ejecución de cinco etapas que se describen a continuación:

Etapa 1. Publicación de la de la actividad: al configurar el Taller en el aula virtual se publicaron una serie de documentos especificando el enunciado de la actividad con la descripción de cada una de las situaciones que debían analizar para desarrollar una propuesta de solución; el proceso de evaluación con la descripción de cada una de las fases de evaluación (autoevaluación, coevaluación y evaluación del profesor), el detalle de los criterios definidos para valorar cada una de los trabajos y la temporalización establecida para cada una de las etapas (entrega del trabajo, revisión y publicación de resultados); y la plantilla de trabajo en la que cada una de las propuestas debe ser desarrollada y entregada. De esta forma se evita que el documento presente detalles que permita identificar el autor.

Etapa 2. Fase de envío: los alumnos de manera individual trabajaron en el análisis de las situaciones y describieron sus propuestas de solución, teniendo como apoyo una plantilla con la descripción de los elementos a incluir. Una vez finalizado el plazo para realizar el envío, se procedió a la asignación de trabajos para la revisión. Se utilizó la asignación aleatoria que ofrece la herramienta Taller, estableciendo como pautas para su configuración un envío por persona y una autoevaluación. De esta forma se intentó evitar en lo posible la presencia de errores sistemáticos que pudieran afectar los resultados, como por ejemplo el sesgo del experimentador. A su vez, se configuró la evaluación para realizar una revisión doble ciego, manteniendo el anonimato de los autores y revisores, con el objetivo de eliminar posibles subjetividades por parte de los alumnos a la hora de evaluar al compañero. En el caso de la evaluación realizada por el profesor fue de simple ciego, ya que conocía el autor del trabajo.

Etapa 3. Fase de evaluación: la revisión de las propuestas se realizó a través del formulario que ofrece la herramienta, con la descripción de los aspectos a valorar, así como también espacios para comentar y ofrecer una retroalimentación formativa de la propuesta. Cada uno de los alumnos participó en la autoevaluación de su trabajo y en la evaluación del trabajo del compañero (evaluación entre iguales). Por su parte, el profesor evaluó igualmente cada uno de los trabajos entregados por los alumnos. Como resultado de la evaluación se obtuvieron tres valoraciones (sumativa y formativa).

Etapa 4. Publicación de resultados y evaluación de la experiencia: una vez finalizada el plazo para llevar a cabo el proceso de revisión, se procedió a publicar los resultados y distribuir un cuestionario utilizando la herramienta de formulario que ofrece Google Drive. Una vez finalizado el plazo para enviar respuestas, se procedió al análisis de los datos recogidos.

\section{RESULTADOS Y DISCUSIÓN}

En primer lugar, se exponen los resultados obtenidos en el análisis factorial, para luego presentar el análisis de fiabilidad del cuestionario y, por último, la prueba Chi cuadrado, permitiendo realizar un análisis de las respuestas de los alumnos. En una primera etapa se lleva a cabo un Análisis Factorial Exploratorio. Como criterio de extracción de los factores se sigue el propuesto por Kaiser: se seleccionan únicamente los factores cuyo valor propio sea igual o superior a 1. De esta forma, se obtuvo una estructura factorial compuesta por 5 factores que explicaban en total un $\mathbf{8 0 . 6 0 6} \%$ de la variabilidad de las puntuaciones de los sujetos.

Como uno de los componentes está integrado por un solo ítem, se decide repetir el análisis fijando en 4 el número de factores a extraer. De esta forma, no aparece ningún factor significativo que se relacione con un único ítem. La medida de adecuación muestral KMO (Kaiser-Meyer-Olkin) arroja un valor de 0.606, que se ve compensado con una elevada significación $(<0.001)$ en la prueba de esfericidad de Bartlett. La interpretación del significado de los factores se lleva a cabo en la matriz rotada por lo que en la tabla 1 se recogen los autovalores y los porcentajes de varianza después de aplicar el procedimiento de rotación de Varimax. Los 4 factores explican el $73.73 \%$ de la varianza.

En la tabla 2 aparecen las saturaciones factoriales más elevadas de cada ítem con cada factor. Los resultados alcanzados facilitan conocer el sentido de los componentes extraídos. Los ítems 1, 2, 4, 5, 7, 9 y 10 se correlacionan de forma elevada con el primer factor extraído. relacionándose con aspectos referidos al proceso de aprendizaje y autorregulación. Los ítems 12 y 15 saturan en el segundo factor, cuyo significado puede ser ventajas del uso de la herramienta. Los ítems 3, 6, 8 y 11 presentan un elevado peso factorial en 
el factor 3 cuyo sentido se relacionará con la Falta de credibilidad como evaluador. El último factor extraído correlaciona con los ítems 13 y 14 por lo que medirá las desventajas del uso de la herramienta.

Tabla 1: Autovalores iniciales.

\begin{tabular}{|l|l|l|l|}
\hline Componente & Autovalor & $\begin{array}{c}\text { \% de la } \\
\text { varianza }\end{array}$ & \% acumulado \\
\hline 1 & 5.931 & 39.542 & 39.542 \\
\hline 2 & 2.090 & 13.935 & 53.478 \\
\hline 3 & 1.929 & 12.861 & 66.338 \\
\hline 4 & 1.109 & 7.392 & 73.730 \\
\hline
\end{tabular}

Tabla 2: Matriz de componentes rotados.

\begin{tabular}{|l|c|c|c|c|}
\hline \multirow{2}{*}{ Ítems } & \multicolumn{3}{|c|}{ Componente } \\
\cline { 2 - 5 } & \multicolumn{1}{|l}{2} & \multicolumn{2}{|c|}{3} & 4 \\
\hline 1. Comprender mejor el contenido de la unidad & .832 & & & \\
\hline 2. Una mayor implicación en el proceso de aprendizaje de la asignatura & .838 & & & \\
\hline 4. Tener una actitud más crítica ante mi propia tarea (producción) & .502 & & & \\
\hline 5. Sentirme mejor valorado/a al considerar mi opinión y no solo la del docente & .712 & & & \\
\hline $\begin{array}{l}\text { 7. Enriquecer la visión individual de los contenidos de la unidad al tener que } \\
\text { profundizar en ellos para evaluar la tarea del compañero }\end{array}$ & .634 & & & \\
\hline 9. Facilita el autoaprendizaje sobre los contenidos de la unidad & .761 & & & \\
\hline $\begin{array}{l}\text { 10. Una experiencia positiva porque posibilita obtener mayor retroalimentación } \\
\text { sobre mi tarea (no sólo la del docente) }\end{array}$ & .638 & & & \\
\hline 12. Facilita la evaluación entre iguales & & .833 & & \\
\hline 15. Ha sido un recurso que favorece mi aprendizaje (valor añadido) & & .862 & & \\
\hline 3. Desempeñar un papel pasivo en el proceso de aprendizaje de la asignatura & & & .734 & \\
\hline 6. Ignorar mis errores en la tarea & & & .795 & \\
\hline 8. Desaprovechar nuevas visiones o diferentes formas de hacer una tarea & & & .539 & \\
\hline $\begin{array}{l}\text { 11. Una experiencia negativa porque las valoraciones de mi compañero no } \\
\text { son útiles para mejorar mi tarea }\end{array}$ & & & .361 & \\
\hline 13. Me ha resultado complicado de usar & & & & .796 \\
\hline $\begin{array}{l}\text { 14. Obstaculiza la implicación de todos los actores (compañeros y docentes) } \\
\text { en el proceso de evaluación }\end{array}$ & & & & .780 \\
\hline
\end{tabular}

Dado que los factores 2 y 4 están integrados solo por dos ítems, y que la solución forzada a dos factores tampoco resulta satisfactoria, se opta por aplicar un Análisis Factorial Confirmatorio (tabla 3) partiendo de la hipótesis de la existencia de unidimensionalidad en la estructura interna del cuestionario. En la tabla 3, donde se muestran las saturaciones factoriales, aparecen cuatro elementos con cargas factoriales inferiores a .40 (ítems $3,6,13$ y 14ㅇ). Sin embargo, su cercanía al valor de referencia y la cuantía de sus índices de homogeneidad (ver tabla 4), apoyan la decisión de mantenerlos en el cuestionario. De esta forma, se cumple la hipótesis de la unidimensionalidad del cuestionario; todos sus elementos contribuyen a medir el mimo constructo. La fiabilidad de la escala se analiza mediante el cálculo del coeficiente alfa de Cronbach, ya que únicamente se trabaja con una sola dimensión, y a partir de los índices de homogeneidad de cada uno de los ítems. El coeficiente de fiabilidad alcanzado es .878.

La fiabilidad alcanzada por la escala es más que satisfactoria. Los índices de homogeneidad de todos los ítems reflejan una elevada relación con la subescala a la que pertenecen ya que superan el valor 0.30 (o quedan muy próximos a este como en el caso del ítem número 14). Todos contribuyen de manera relevante a la fiabilidad de la dimensión a la que pertenecen. Las respuestas de los alumnos a los diferentes ítems de la escala se analizan mediante la prueba no paramétrica Chi cuadrado de Pearson ( $\mathrm{x} 2$ ). Este método comprueba si hay diferencias significativas en las frecuencias de elección de cada una de las categorías mediante las que se podía contestar a los ítems. Cuando el estadístico de contraste de la prueba X2 es igual a 0 , las frecuencias de las categorías no son significativamente diferentes. Si existe una diferencia cualquiera 
entre las frecuencias, aparecerá un $\mathrm{X} 2$ superior a 0 , valor que irá aumentando si no es producto del azar, hasta alcanzar valores significativos que permitan rechazar la hipótesis nula de la igualdad entre la teoría y la realidad. La tabla 5 recoge los resultados de la prueba obtenidos para cada uno de los ítems. En todos los ítems las diferencias encontradas son significativas con valores, en la mayoría de los casos, inferiores a 0.01.

Tabla 3: Matriz de componentes.

\begin{tabular}{|l|c|}
\hline \multicolumn{1}{|c|}{ Ítems } & Componente \\
\cline { 2 - 2 } & 1 \\
\hline 1. Comprender mejor el contenido de la unidad & .715 \\
\hline 2. Una mayor implicación en el proceso de aprendizaje de la asignatura & .686 \\
\hline 3. Desempeñar un papel pasivo en el proceso de aprendizaje de la asignatura & .374 \\
\hline 4. Tener una actitud más crítica ante mi propia tarea (producción) & .686 \\
\hline 5. Sentirme mejor valorado/a al considerar mi opinión y no solo la del docente & .620 \\
\hline 6. Ignorar mis errores en la tarea & .351 \\
\hline $\begin{array}{l}\text { 7. Enriquecer la visión individual de los contenidos de la unidad al tener que profundizar en ellos } \\
\text { para evaluar la tarea del compañero }\end{array}$ & .843 \\
\hline 8. Desaprovechar nuevas visiones o diferentes formas de hacer una tarea & .564 \\
\hline 9. Facilita el autoaprendizaje sobre los contenidos de la unidad & .887 \\
\hline $\begin{array}{l}\text { 10. Una experiencia positiva porque posibilita obtener mayor retroalimentación sobre mi tarea (no } \\
\text { sólo la del docente) }\end{array}$ & .840 \\
\hline $\begin{array}{l}\text { 11. Una experiencia negativa porque las valoraciones de mi compañero no son útiles para } \\
\text { mejorar mi tarea }\end{array}$ & .569 \\
\hline 12. Facilita la evaluación entre iguales & .664 \\
\hline 13. Me ha resultado complicado de usar & .379 \\
\hline $\begin{array}{l}\text { 14. Obstaculiza la implicación de todos los actores (compañeros y docentes) en el proceso de } \\
\text { evaluación }\end{array}$ & .302 \\
\hline 15. Ha sido un recurso que favorece mi aprendizaje (valor añadido) & .558 \\
\hline
\end{tabular}

Tabla 4: Índice de homogeneidad de los ítems.

\begin{tabular}{|l|c|}
\hline \multicolumn{1}{|c|}{ Ítems } & $\mathrm{IH}$ \\
\hline 1. Comprender mejor el contenido de la unidad & .593 \\
\hline 2. Una mayor implicación en el proceso de aprendizaje de la asignatura & .588 \\
\hline 3. Desempeñar un papel pasivo en el proceso de aprendizaje de la asignatura & .321 \\
\hline 4. Tener una actitud más crítica ante mi propia tarea (producción) & .593 \\
\hline 5. Sentirme mejor valorado/a al considerar mi opinión y no solo la del docente & .515 \\
\hline 6. Ignorar mis errores en la tarea & .313 \\
\hline $\begin{array}{l}\text { 7. Enriquecer la visión individual de los contenidos de la unidad al tener que } \\
\text { profundizar en ellos para evaluar la tarea del compañero }\end{array}$ & .770 \\
\hline 8. Desaprovechar nuevas visiones o diferentes formas de hacer una tarea & .516 \\
\hline 9. Facilita el autoaprendizaje sobre los contenidos de la unidad & .808 \\
\hline $\begin{array}{l}\text { 10. Una experiencia positiva porque posibilita obtener mayor retroalimentación } \\
\text { sobre mi tarea (no sólo la del compañero) }\end{array}$ & .759 \\
\hline
\end{tabular}


Tabla 4 (continuación)

\begin{tabular}{|l|c|}
\hline $\begin{array}{l}\text { 11. Una experiencia negativa porque las valoraciones de mi compañero no son } \\
\text { útiles para mejorar mi tarea }\end{array}$ & .488 \\
\hline 12. Facilita la evaluación entre iguales & .605 \\
\hline 13. Me ha resultado complicado de usar & .372 \\
\hline $\begin{array}{l}\text { 14. Obstaculiza la implicación de todos los actores (compañeros y docentes) en el } \\
\text { proceso de evaluación }\end{array}$ & .297 \\
\hline 15. Ha sido un recurso que favorece mi aprendizaje (valor añadido) & .501 \\
\hline
\end{tabular}

Tabla 5: Resultados de la prueba de Chi cuadrado. (Prueba Chi-Cuadrado * $p \leq .05 ;{ }^{* *} p \leq .01$ )

\begin{tabular}{|c|c|c|c|}
\hline Ítem & Media & $\begin{array}{l}\text { Desviación } \\
\text { típica }\end{array}$ & $\begin{array}{c}\text { Chi } \\
\text { cuadrado }\end{array}$ \\
\hline 1. Comprender mejor el contenido de la unidad & 3.71 & .938 & $15.706^{\star *}$ \\
\hline 2. Una mayor implicación en el proceso de aprendizaje de la asignatura & 3.85 & .925 & $17.471^{\star \star}$ \\
\hline $\begin{array}{l}\text { 3. Desempeñar un papel pasivo en el proceso de aprendizaje de la } \\
\text { asignatura }\end{array}$ & 1.85 & .857 & $26.882^{\star *}$ \\
\hline 4. Tener una actitud más crítica ante mi propia tarea (producción) & 4.18 & .869 & $26.588^{* *}$ \\
\hline $\begin{array}{l}\text { 5. Sentirme mejor valorado/a al considerar mi opinión y no solo la del } \\
\text { docente }\end{array}$ & 3.79 & 1.149 & $12.176^{*}$ \\
\hline 6. Ignorar mis errores en la tarea & 1.53 & .788 & $44.235^{\star *}$ \\
\hline $\begin{array}{l}\text { 7. Enriquecer la visión individual de los contenidos de la unidad al tener que } \\
\text { profundizar en ellos para evaluar la tarea del compañero }\end{array}$ & 3.94 & .851 & $32.176^{\star *}$ \\
\hline 8. Desaprovechar nuevas visiones o diferentes formas de hacer una tarea & 1.79 & .880 & $25.706^{\star \star}$ \\
\hline 9. Facilita el autoaprendizaje sobre los contenidos de la unidad & 4.03 & .870 & $22.765^{\star *}$ \\
\hline $\begin{array}{l}\text { 10. Una experiencia positiva porque posibilita } \\
\text { retroalimentación sobre mi tarea (no sólo la del docente) }\end{array}$ & 4.06 & 1.153 & $26.294^{\star *}$ \\
\hline $\begin{array}{l}\text { 11. Una experiencia negativa porque las valoraciones de mi compañero no } \\
\text { son útiles para mejorar mi tarea }\end{array}$ & 1.65 & .849 & $38.059^{* *}$ \\
\hline 12. Facilita la evaluación entre iguales & 3.88 & .880 & $31.882^{* *}$ \\
\hline 13. Me ha resultado complicado de usar & 1.94 & .919 & $19.824^{* *}$ \\
\hline $\begin{array}{l}\text { 14. Obstaculiza la implicación de todos los actores (compañeros y docentes) } \\
\text { en el proceso de evaluación }\end{array}$ & 1.97 & 1.000 & $19.529^{* *}$ \\
\hline 15. Ha sido un recurso que favorece mi aprendizaje (valor añadido) & 3.85 & .892 & $28.353^{\star *}$ \\
\hline
\end{tabular}

Los valores de las medias de los ítems expuestos en la Tabla 5 permiten observar la actitud positiva del grupo con respecto a la evaluación por pares. De acuerdo con los resultados (media del ítem 10: 4.06), se evidencia la importancia que tiene la retroalimentación recibida, proveniente no solo del docente, para comprender mejor el contenido y tener una actitud crítica sobre su tarea. Asimismo, los encuestados opinan mayoritariamente que facilita el autoaprendizaje de los contenidos (media del ítem 9: 4.03). Por el contrario, los ítems que reflejan actitudes negativas hacia la evaluación por pares son los que obtienen valores promedio más bajos (ítems $3,6,8,11,13$ y 14, con medias inferiores a 2 puntos).

\section{DISCUSIÓN FINAL}

Los resultados aportados posibilitan la replicación del estudio de cara a la obtención de evidencias empíricas que apoyen las ventajas que aporta el uso del Taller de Moodle para la evaluación entre iguales. Las diferencias significativas entre las elecciones realizadas por los estudiantes a las diferentes categorías de los ítems refuerzan la calidad de los datos recogidos. Los promedios de los ítems evidencian la favorable actitud del grupo hacia la evaluación por pares, al igual que el impacto positivo que tiene este tipo de experiencias para el proceso de aprendizaje y el desarrollo de competencias relacionadas con la evaluación, tal como señalan otros estudios (Siow, 2015). Los indicadores destacan como el alumnado está de acuerdo en que deben tener un papel activo en el proceso de aprendizaje de la asignatura y que no pueden desaprovechar la retroalimentación de sus compañeros, que les permita obtener otra visión sobre su tarea, identificar errores y mejorarlos. Estas percepciones concuerdan con los resultados de otros estudios, donde la evaluación del compañero es una práctica bien aceptada y en las que no se desaprueban las competencias que posee el alumnado como evaluadores (Siow, 2015; 2011; Altinay, 2017; Fernandes et al., 2016; Contreras, 2018). 
A su vez, el alumnado estuvo de acuerdo en que el Taller de Moodle, como herramienta, facilita el proceso de evaluación, convirtiéndose en un recurso que favorece su aprendizaje. El hecho de que muestren actitudes positivas hacia el uso de esta es un aspecto importante, ya que puede garantizar la obtención de mayores beneficios, como la generación de nuevos aprendizajes. No obstante, a pesar de que los alumnos no señalan desventajas sobre la herramienta, como la dificultad de uso o que pueda obstaculizar el proceso de evaluación, se recomienda para futuros estudios añadir otros ítems que permitan profundizar en posibles limitaciones. Al igual que incorporar otros indicadores para analizar en aspectos que pueden influir en la percepción que tiene el alumnado al ejercer funciones de evaluador y aceptar juicios al ser evaluado por un compañero como, la comprensión de los criterios de evaluación, experiencia como evaluador y confianza a la hora de realizar juicios y valoraciones (Nortcliffe, 2012; Ashenafi, 2017).

Por otra parte, de acuerdo con los resultados expuestos, se puede evidenciar como existen razones para involucrar al alumnado en los procesos de evaluación haciendo uso de herramientas tecnológicas como el Taller de Moodle. Aprendemos mostrando y articulando con otros lo que sabemos (Liu y Carless, 2006), por lo tanto, en el caso de la educación online, el poder desarrollar experiencias como esta permitirá que los alumnos puedan explorar, participar activamente e intercambiar criterios, mejorando su aprendizaje

De acuerdo con lo planteado y teniendo en cuenta las posibilidades que ofrece el Taller de Moodle, no es de extrañar que los resultados recogidos en esta experiencia muestren las actitudes positivas del alumnado ante el uso de la herramienta para la evaluación entre iguales, ya que, además de facilitar el proceso, el profesor puede mediar y guiar la experiencia, para garantizar el logro de los objetivos propuestos. Entre las ventajas que se lograron identificar del Taller de Moodle y que pueden apoyar lo argumentado, se pueden destacar que permite el envío de archivos con distintos formatos y se puede configurar para realizar una revisión doble ciego, manteniendo el anonimato de los autores y revisores, con el objetivo de eliminar posibles subjetividades por parte de los alumnos a la hora de evaluar al compañero.

Además, se pueden llevar a cabo de forma paralela distintos modelos de evaluación: autoevaluación, evaluación entre iguales y evaluación del profesor, y el alumno puede disponer de información sobre los criterios de evaluación al igual que ejemplos para practicar la evaluación. Esta posibilidad puede brindar mayor seguridad al alumno si no ha participado en otras experiencias como evaluador. A su vez, la asignación de trabajos se puede hacer de forma manual o aleatoria, evitando en lo posible la presencia de errores sistemáticos que pudieran afectar los resultados. Por último, ofrece cuatro estrategias de calificación (calificación acumulativa, comentarios, número de errores y rúbrica). Esto permite experimentar y aplicar distintas estrategias para valorar los resultados obtenidos en la evaluación por pares.

\section{CONCLUSIONES}

De acuerdo con el trabajo presentado y a los resultados obtenidos, se pueden plantear las siguientes conclusiones principales:

1. Se recomienda la evaluación entre iguales como método alternativo en la educación superior online, ya que complementa la evaluación del profesor e involucra de forma activa al alumnado, fomentando la responsabilidad y la implicación en su propio proceso de aprendizaje (Siow, 2015; Kishwar, et al., 2015). Como, por ejemplo, involucrar su participación en la redacción y descripción de los criterios de evaluación.

2. La mediación del docente es fundamental. Por ello la capacitación debe estar dirigida a mejorar las prácticas desarrolladas actualmente, con el objetivo de que exista relación entre las propuestas que se desarrollan en los entornos virtuales y la evaluación de los aprendizajes. No se pueden trabajar como dos elementos separados, ya que ambos forman parte del proceso didáctico. Desde esta perspectiva la tecnología continuará estando al servicio de la enseñanza.

3. La tecnología facilita los procesos de evaluación entre iguales, a través de herramientas como el Taller de Moodle. No se trata de pruebas automatizadas, sino de sistemas que promueven una evaluación formativa, teniendo como eje motor la retroalimentación, así como el análisis y la reflexión de la práctica educativa. Por lo tanto, se deben continuar planificando y diseñando experiencias como las presentadas en este estudio, procurando una evaluación del aprendizaje, para el aprendizaje y desde el aprendizaje (Barberà, 2016).

\section{REFERENCIAS}

Acedo, M.A., y Ruiz-Cabestre, F.J., An experience about autonomous or participative assessment: Self assessment and peer assessment, https://doi.org/10.3989/arbor.2011.extra-3n3142, Arbor, 187(3), 183-188 (2011).

Altınay, Z., Evaluating peer learning and assessment in online collaborative learning environments, https://doi.org/10.1080/0144929x.2016.1232752, Behaviour and Information Technology, 36(3), 312-320 (2017). 
Ashenafi, M.M., Peer-assessment in higher education - twenty-first century practices, challenges and the way forward, https://doi.org/10.1080/02602938.2015.1100711, Assessment and Evaluation in Higher Education, 42(2), 226-251 (2017).

Barberà, E., Aportaciones de la tecnología a la e-Evaluación, https://doi.org/10.6018/red/50/4, RED Revista de Educación a Distancia, 50(4), 1-10 (2016).

Cassady, J. y Gridley, E., The Effects of Online Formative and Summative Assessment on Test Anxiety and Performance, The Journal of Technology, Learning, and Assessment, 4(1), 1-31 (2005).

Cook, D.A., y Ellaway, R.H., Evaluating technology-enhanced learning: $A$ comprehensive framework, https://doi.org/10.3109/0142159x.2015.1009024, Medical Teacher, 37(10), 961-970 (2015).

Contreras, G.A., Retroalimentación por Pares en la Docencia Universitaria. Una Alternativa de Evaluación Formativa, https://doi.org/10.4067/s0718-50062018000400083, Formación universitaria, 11(4), 83-94 (2018).

Cordero, J.A. y Caballero, A., The Moodle platform: A useful tool for training in life support. Analysis of satisfaction questionnaires from students and instructors of the semFYC advanced life support courses, https://doi.org/10.1016/j.aprim.2015.02.006, Atención Primaria, 47(6), 376-384 (2015).

De La Hoz, L.P., Acevedo, D. y Torres. J., Uso de Redes Sociales en el Proceso de Enseñanza y Aprendizaje por los Estudiantes y Profesores de la Universidad Antonio Nariño, Sede Cartagena, https://doi.org/10.4067/s071850062015000400009, Formación Universitaria, 8(4), 77-84 (2015).

Deepak, K.C., Evaluation of Moodle features at Kajaani University of Applied Sciences-Case Study, https://doi.org/10.1016/j.procs.2017.10.021, Procedia Computer Science, 116, 121-128 (2017).

Fernandes, D.J., Sotolongo, M. y Martínez, C.C., La Evaluación del Desempeño por Competencias: Percepciones de Docentes y Estudiantes en la Educación Superior, https://doi.org/10.4067/s0718-50062016000500003, Formación Universitaria, 9(5), 15-24 (2016).

Gallego, B., Quesada, V., Gómez M.A., y Cubero, J., La evaluación y retroalimentación electrónica entre iguales para la autorregulación $y$ el aprendizaje estratégico en la universidad: la percepción del alumnado, https://doi.org/10.4995/redu.2017.5991, Revista de Docencia Universitaria, 15(1), 127-146 (2017).

Guardia, L. y Sangrá, A., Diseño instruccional y objetos de aprendizaje: hacia un modelo para el diseño de actividades de evaluación del aprendizaje on-line. RED Revista de Educación a Distancia, 4, 1-14 (2005).

Ho, V.W., Harris, P.G., Kumar, R.K., y Velan, G.M., Knowledge maps: a tool for online assessment with automated feedback, https://doi.org/10.1080/10872981.2018.1457394, Medical Education Online, 23(1), 1-9 (2018).

Ibarra, M.S. Rodríguez, G. y Gómez, M.A., La evaluación entre iguales: beneficios y estrategias para su práctica en la universidad, Revista de Educación, 359, 206-231 (2012).

Jenaro, C., Castaño, R., Martín, M.E, y Flores, N.E., Academic performance in higher education and its association to active participation in the moodle platform, Estudios Sobre Educacion, 34, 177-198 (2018).

Kiliç, D., An Examination of Using Self-, Peer-, and Teacher-Assessment in Higher Education: A Case Study in Teacher Education, https://doi.org/10.5539/hes.v6n1p136, Higher Education Studies, 6(1), 136-144 (2016).

Kishwar, M., Ahmad, A., Sheikh, S., ul Haque Sheikh, S., y llyas, J., Peer Assessment: An Innovative Tool for Assessment in Higher Education. Pakistan Armed Forces Medical Journal, 65(2), 268-272 (2015).

Liu, M.F. y Carless, D., Peer feedback: The learning element of peer assessment, https://doi.org/10.1080/13562510600680582, Teaching in Higher Education, 11(3), 279-290 (2006).

Nortcliffe, A., Can Students Assess Themselves and Their Peers? - A Five Year Study, https://doi.org/10.7190/seej.v1i2.29, Student Engagement and Experience Journal, 1(2) (2012).

Obura, B., Agak, J, Odhiambo, K., y Kiprotich, P., Student Perceptions of the Effectiveness of Formative Assessment in an Online Learning Environment. https://doi.org/10.5944/openpraxis.10.1.705, Open Praxis, 10(1), 29-39 (2018).

Siow, L.F., Students' Perceptions on Self- and Peer-Assessment in Enhancing Learning Experience. Malaysian Online Journal of Educational Sciences, 3(2), 21-35 (2015).

Strang, K.D., Effectiveness of peer assessment in a professionalism course using an online workshop, https://doi.org/10.28945/2100, Journal of Information Technology Education: Innovations in Practice, 14, 1-16 (2015).

Thawabieh, A.M., A Comparison between Students' Self-Assessment and Teachers' Assessment, https://doi.org/10.5430/jct.v6n1p14, Journal of Curriculum and Teaching, 6(1), 14-20 (2017).

Troy, T. y Bulgakov-Cooke, D., Formative Assessment with Technology 2011-12: Second Year of Implementation. Eye on Evaluation, D\&A Report, 13(5), 1-30 (2013).

Wang, K.H., Wang, T., Wang, W.L., y Huang, S., Learning styles and formative assessment strategy: enhancing student achievement in Web-based learning, https://doi.org/10.1111/j.1365-2729.2006.00166.x, Journal of Computer Assisted Learning, 22(3), 207-217 (2006). 